\title{
TTR
}

Traduction, terminologie, re?daction

\section{Traducteurs médiévaux, traductrices féministes : une même éthique de la traduction?}

\section{Jean Delisle}

Volume 6, numéro 1, 1er semestre 1993

L'Histoire en traduction

URI : https://id.erudit.org/iderudit/037144ar

DOI : https://doi.org/10.7202/037144ar

Aller au sommaire du numéro

Éditeur(s)

Association canadienne de traductologie

ISSN

0835-8443 (imprimé)

1708-2188 (numérique)

Découvrir la revue

Citer cet article

Delisle, J. (1993). Traducteurs médiévaux, traductrices féministes : une même éthique de la traduction? TTR, 6(1), 203-230. https://doi.org/10.7202/037144ar

Tous droits réservés (C) TTR: traduction, terminologie, rédaction — Les auteurs, 1993
Ce document est protégé par la loi sur le droit d'auteur. L'utilisation des services d'Érudit (y compris la reproduction) est assujettie à sa politique d'utilisation que vous pouvez consulter en ligne.

https://apropos.erudit.org/fr/usagers/politique-dutilisation/ 


\section{Traducteurs médiévaux, traductrices féministes: une même éthique de la traduction?}

\section{Jean Delisle}

Il peut sembler étonnant, voire incongru, de tenter de tracer un parallèle entre les traducteurs du Moyen Âge français et les traductrices féministes canadiennes. Que peuvent bien avoir en commun Jean de Meung, Pierre Bersuire, Raoul de Presles, Nicole Oresme, Simon de Hesdin, Laurent de Premierfait, Jean Miélot et les traductrices Susanne de Lotbinière-Harwood, Barbara Godard, Kathy Mezei, Marlene Wildeman, Fiona Strachan, Yvonne Klein, Luise von Flotow?

De prime abord, tout semble séparer ces deux groupes d'artisans de la traduction, qui appartiennent à des univers culturels totalement différents. Au Moyen Âge, ce sont essentiellement des hommes qui s'adonnent à cette activité. Ils sont moines, religieux, avocats, "valets de chambre», médecins, professeurs. La vie intellectuelle et artistique gravite autour des monastères et des cours seigneuriales. Les cuvres traduites, généralement commandées par le roi ou des seigneurs lettrés, ont toutes un caractère utilitaire. Ce qui intéresse l'élite d'alors, ce sont les traités politiques, économiques, historiques, astronomiques, techniques, en un mot, les ouvrages pratiques. On traduit surtout les auctoritates: grands auteurs de l'Antiquité grécoromaine, savants et théologiens réputés, docteurs de l'Église. Le roi, qui ne veut pas être un "âne couronné», veille à sa propre 
instruction, et souhaite élever le niveau intellectuel des gens de sa cours. Plusieurs traducteurs sont aussi précepteurs des grands du royaume. Le public lecteur se compose, outre le roi lui-même, des hauts dignitaires civils et ecclésiastiques, des lettrés et des nobles dames qui, ignorant le latin, passent commande à leur «latinier» de leur bailler tel ouvrage en langue vulgaire. Rappelons que la montée des langues vulgaires en Europe s'est faite concurremment à une intensification de la connaissance du latin. Dans les monastères et chez les érudits, de même qu'au sein des appareils administratifs et judiciaires, le latin garde la prééminence, et le pouvoir est solidement détenu par les hommes. Dans le domaine qui nous intéresse, on peut compter sur les doigts de la main les femmes qui pratiquent la traduction, et dont les noms sont parvenus jusqu'à nous.

Par contraste, aujourd'hui, au nombre des adeptes de l'approche féministe de la traduction on compte presque essentiellement des femmes. A notre connaissance, le seul homme à se définir comme traducteur féministe est Howard Scott, traducteur de l'Euguélionne (Louky Bersianik) et de Antre (Madeleine Gagnon). Contrairement aux traducteurs du Moyen Âge qui travaillaient de façon plus ou moins isolée, les traductrices canadiennes se connaissent toutes, se rencontrent fréquemment dans des colloques ou lors de tables rondes et publient, conjointement avec les écrivaines qu'elles traduisent, des collectifs ou des numéros thématiques de revues comme Tessera ${ }^{1}$. Ces rencontres et ces publications donnent lieu à une réflexion originale, intense, «avant-gardiste» même, sur la traduction. Les ouvrages traduits se composent presque essentiellement d'œuvres littéraires: romans, nouvelles, essais, poésie, articles ou textes de conférence. En outre, ces écrits ont en commun d'être liés à une idéologie précise: le féminisme. On peut donc dire que les féministes s'entre-traduisent. Fait important à signaler, la majorité des traductions se font du français vers l'anglais, les romancières, poètes ou théoriciennes féministes québécoises étant traduites par leurs collègues canadiennes-anglaises.

1. Cf. David Homel et Sherry Simon, dir. (1988), p. 48. 
Au-delà de ces divergences évidentes - et il serait facile d'allonger la liste -, il est possible de dégager néanmoins plusieurs points communs entre la manière de traduire des traducteurs médiévaux français et celle des traductrices féministes canadiennes. Nous avons fait porter notre rapprochement sur les cinq points suivants:

1. Appropriation du texte de départ

2. Recherche d'une légitimité

3. Schéma et didactisme des préfaces

4. Interventions sur la langue

5. Visibilité du traducteur ou de la traductrice dans sa traduction.

\section{Appropriation du texte de départ}

A propos de la manière de traduire des traducteurs du Moyen Âge, Jacques Monfrin (1964a) écrit:

Il semble bien qu'on ait rarement eu, avant la fin du Moyen Âge, le souci historique et philologique de laisser ou de retrouver l'œuvre $d$ 'un auteur sous la forme exacte que celui-ci avait voulu lui donner. Suivant une idée généralement répandue, tout écrit destiné à instruire est perfectible et du moment qu'on le transcrit et qu'on le traduit, on ne voit aucune raison pour ne pas le modifier au goât du jour ou l'améliorer en le complétant à l'aide de renseignements puisés à d'autres sources. (pp. 217-218)

Tout écrit destiné à instruire est perfectible. Pour les traducteurs médiévaux comme pour les traductrices contemporaines, le texte n'apparaît pas comme un donné immuable qu'il faut respecter, voire vénérer, mais comme une matière première que l'on peut réaménager plus ou moins à sa guise, comme le prône Kathy Mezei (1989):

To translate is to invent, create, and often to betray-the source. Translation is a daring act, one that requires courage and faith, and women who write are especially attuned to writing as translation for not only must we translate our 
'source,' but we must decide whether to translate into the dominant discourse, the accepted discourse of patriarchy, the 'androlect,' or instead to venture forth into another language that seems to have to be transcribed as we go. (p. 9)

Dans la perspective féministe, la traduction est production, et non simple re-production de sens. C'était aussi un des traits caractéristiques de la façon de traduire de leurs lointains prédécesseurs.

Aux yeux des féministes, la langue n'est pas neutre. Façonnée par l'homme, elle porte l'empreinte de son pouvoir, de sa vision du monde et de la place (subalterne) qu'il réserve à la femme. Les féministes refusent donc de "parler masculin» et s'efforcent de «parler femme». Elles entretiennent la conviction que celui ou celle qui détient les connaissances détient aussi le pouvoir. Et la parole est instrument de pouvoir, même la parole relayée par la traduction. Susanne de Lotbinière-Harwood (1991) l'exprime on ne peut plus clairement:

[...] loin d'être neutre, l'acte de traduire constitue une prise de parole [...]. En plus d'être une voie de passage d'une langue à une autre, la traduction est aussi un lieu de pouvoir. Pour les traductrices féministes, elle représente un espace à investir, un pouvoir à exercer. (p. 12)

Mais cette prise de parole par le traducteur ou la traductrice suppose une opération d'appropriation du texte original. Les artisans de la traduction aux deux époques mises en parallèle ne s'effacent pas devant l'auteur traduit. Ils s'investissent tout entier dans le texte traduit, s'interposent entre l'auteur et le lecteur. Qui plus est, ils dédoublent l'auteur, deviennent coauteurs. Le détournement du texte original, car c'est bien de cela qu'il s'agit, se manifeste concrètement par des interventions du traducteur ou de la traductrice qui normalement sont réservées aux auteurs. Nous en donnerons de nombreux exemples dans la suite de cette étude.

\section{Recherche d'une légitimité}

Au Moyen Âge, le latin est la langue de l'Université, de la science, de l'Église et des actes officiels de l'État. La langue française, quant 
à elle, est encore en gestation. Forts de l'appui des rois successifs, les traducteurs soulèvent une question de principe, celle de la légitimité de la traduction en langue vulgaire. Est-il bon et souhaitable de traduire en langue vulgaire? Cette question fera couler beaucoup $d^{\prime}$ encre et sera au coeur de plusieurs querelles qui se prolongeront jusqu'au milieu du XVI ${ }^{e}$ siècle et même au-delà. Le débat entourant la traduction et l'avènement d'une littérature nationale se cristallisera alors autour du manifeste de Joachim du Bellay, la Deffence et illustration de la langue francoyse (1549).

Jean le Bon (1319-1364), par ses nombreuses commandes de traduction, inaugure une véritable politique de la traduction que continuera d'appliquer son successeur, Charles V, dit le Sage (13371380). Ces rois s'entourent de traducteurs-conseillers et les chargent de «translater pour le bien commun». Nicole Oresme (v. 1320-1382) confirme l'intention des têtes dirigeantes quand il écrit: "sont pluseurs gens de langue françoise qui sont de grant entendement et de excelleent enging et qui n'entendent pas souffisanment latin, et pour ce les vaillans roys de France ont fait aucuns livres translater en françois»"

Dans le prologue de sa traduction des Éthiques d'Aristote, Oresme, se faisant le porte-parole officieux de Charles $V$, réaffirme la politique linguistique du roi en même temps que la légitimité de la traduction: "translater telz livres en françois et baillier en françois les arts et les sciences est un labeur moult proffitable, car c'est un langage noble et commun a genz de grant engin et de bonne prudence" (Ibid., pp. 231-232). Dans son argumentation en faveur du français, le traducteur rappelle qu'autrefois "c'estait bien de translater les sciences de grec en latin", et que maintenant le français peut à son tour translater le latin. Les traducteurs cherchent donc à faire accéder le français à un niveau de langue permettant d'accueillir les auctoritates, à doter l'idiome français du registre de "langue savante», registre qu'on ne reconnaissait alors qu'au latin. Dans le contexte médiéval, l'entreprise de traduction en langue vulgaire apparaît comme une rupture à la base de laquelle il y a une double volonté: "volonté du roi de pouvoir lire dans sa langue les grands textes latins et volonté du traducteur de forcer la langue vernaculaire

2. Cité par Jacques Monfrin (1964a), p. 229. 
à acquérir un registre complètement neuf d'expression" (S. Lusignan, 1986, p. 140). En légitimant la pratique des traductions en français, le roi augmente par le fait même son pouvoir, car les traductions lui fournissent les «armes de l'argumentation", un «support idéologique» (Ibid., p. 134). Ce faisant, il dépouille les clercs d'une partie de leur pouvoir. La traduction revêt, dans ce contexte, la valeur d'un geste éminemment politique.

Les traductrices féministes cherchent elles aussi à établir une légitimité: celle de la parole féminine en regard du discours «patriarcal». Susanne de Lotbinière-Harwood a exposé leur projet collectif dans son ouvrage Re-Belle et Infidele (1991), véritable manifeste, dont l'esprit n'est pas sans rappeler celui de Du Bellay:

[...] la traduction comme pratique de réécriture au féminin met cartes sur table dès le départ. Son projet est de faire entrer la conscience féministe dans l'activité traductive. Comme l'écriture au féminin, dont elle est tributaire, la traduction au féminin se présente comme une activité politique visant à faire apparaître et vivre les femmes dans la langue et dans le monde. (p. 11)

Cet ambitieux projet socio-politico-linguistique est orienté vers l'établissement d'une culture féminine au sens très large du terme. Il s'exprime concrètement par l'affranchissement du discours dominant, de l'ordre symbolique patriarcal qui tend à occulter le féminin. Il se matérialise aussi par la dénonciation de l'exclusion des femmes dans la langue. Il est un effort pour réinvestir la sensibilité féminine, la vision féminine dans le discours littéraire et le quotidien. Le féminisme, que François Ricard (1992) considère comme «la grande idéologie de l'époque» (p. 205), est un filtre qui permet une lecture particulière de la réalité.

Les traductrices féministes revendiquent le droit de prendre la parole, de créer du sens en faisant éclater le caractère "androcentrique» de la langue. Pour paraphraser le titre de l'ouvrage de Patricia Smart, elles font la difficile expérience de Traduire dans la maison du père, c'est-à-dire de tisser l'identité de la femme dans le langage, de reprendre possession des mots, 
d'inscrire le corps dans le dire. Pour ce faire, elles exploitent les glissements de sens que permet le passage de l'écriture à la traduction (réécriture), elles s'autorisent de multiples transgressions, instaurent une nouvelle praxis de la langue. Elles produisent un sens nouveau par de subtiles manipulations textuelles. Alors que les traducteurs du Moyen Âge travaillaient à doter la langue française d'un registre savant, les traductrices féministes cherchent, quant à elles, à doter la langue contemporaine d'un registre proprement féminin. Au sujet de sa traduction de l'œuvre de Nicole Brossard (la Lettre aérienne), Marlene Wildeman (1989) écrit: «Perhaps the greatest translation challenge arose from the fact that The Aerial Letter re-creates, poetically, lesbian feminist consciousness in a (patriarchal) language foreign to it." (pp. 32-33)

Il s'agit là d'une pratique émancipatoire qui se situe aux antipodes de la mimesis. Le texte original n'est pas reproduit selon les règles classiques et orthodoxes de la recherche d'équivalences de même poids sémantique, mais il devient "pré-texte" (prétexte) à un autre discours ${ }^{3}$ qui se veut à la fois semblable et différent. Dans ce texte pris en otage, le sujet traduisant est présent de façon explicite et il y affirme les valeurs féminines et féministes. Dans cette perspective, traduire devient «l'acte d'une subjectivité à l'œuvre dans un contexte socio-politique précis. Le je qui traduit inscrit son savoir, ses choix, ses intentions, ses convictions dans le texte qui se réécrit. La traduction peut donc être un véritable outil politique» (S. de Lotbinière-Harwood, 1991, p. 27). La grille de lecture nouvelle proposée par ces traductrices engagées fait voir des réalités cachées par et dans les mots. Les traductions deviennent porteuses de la pensée féministe et les traductrices, cocréatrices de l'œuvre traduite. L'œuvre originale est jugée perfectible, ou tout au moins remodelable, comme le pensaient aussi les traducteurs du Moyen Âge.

3. «As first reader of the text, reader from a foreign culture, I must abscond with it, hijack it into my own." Barbara Godard (1982), p. 36. 
On ne peut s'empêcher de voir dans l'expression «traductrice féministe» une sorte de contradiction apparente dans les termes, un oxymoron, car, par définition (par tradition?), un traducteur est privé du droit à la parole (quand il réexprime celle d'un auteur), tandis qu'une féministe est, par définition, une militante qui prend la parole afin de donner une voix aux femmes. La traductrice féministe s'arroge donc le droit de rétrécir, voire de supprimer l'écart qui existe entre la langue «maternelle» et la langue-du-père dans une vaste et difficile opération de «transformance»".

En somme, le combat de la légitimité, les traductrices féministes le mènent sur plusieurs fronts simultanément: sur le plan politique (briser l'hégémonie de la langue patriarcale $e^{5}$ ), féministe (affirmer la place qui revient à la femme dans la société et dénoncer tous les codes et conventions qui la maintiennent dans un état de dépendance), sur le plan éthique (la traductrice se définit comme coauteure, sort de l'ombre, se libère de sa chape d'anonymat). De même que les féministes réclament l'égalité des droits dans tous les domaines de la vie sociale, les traductrices engagées réclament un droit de parole identique à celui dont jouit l'auteur - homme ou femme - des textes originaux traduits. Elles instaurent une nouvelle dynamique auteuretraductrice et redéfinissent la notion de fidélité en traduction. Elles s'emploient à rendre explicite ce qui est implicite, à écrire «l'inédit», mot fétiche de Nicole Brossard.

4. "Transformance" is also the collective title for the re/writing (translation) project in which Nicole Brossard has been involved with Daphne Marlatt. Brossard's activities of transformance stand as a model for feminist discourse/translation in its actions to re/reading and re/writing, its dialogism." Barbara Godard (1989), p. 46.

5. Des organismes publics et des entreprises privées ont déjà adopté des politiques en matière de féminisation. Les rédactrices et les rédacteurs à leur service sont tenus de les appliquer. 
It seems evident that in devising and practising creative non-traditional approaches to translation, making their presence felt in the texts and challenging their authors, feminist translators in Canada are making changes to some of these traditional views and the habitual "missionary" position assigned to translation. (Luise von Flotow, 1991, p. 69)

\section{Schéma et didactisme des préfaces}

Les préfaces des traducteurs remplissent diverses fonctions, mais on peut dire à la suite de Sherry Simon (D. Homel et S. Simon, 1988) que, de façon générale, «a successful preface draws out the complex links between language, culture and the particular destiny that is desired for the literary work» (p. 53). L'œuvre originale devant normalement se suffire à elle-même, les préfaces ont donc toujours un caractère didactique.

Le Moyen Âge est l'une des périodes de l'histoire de la traduction où le rôle de vulgarisateur et de pédagogue joué par les traducteurs est le plus évident. «Inevitably the function of servicing an unlettered public implied at first a degree of didacticism, and the conception of translation as a teaching activity was never more apt.» (J. Beer, 1989, p. 1) Les traducteurs de cette époque usent couramment des possibilités que leur offrent les prologues, "prohesmes", préfaces et notes pour ajouter aux textes originaux un supplément d'information. Les traductrices contemporaines font elles aussi un grand usage de ces éléments péritextuels. Le «détournement» qui consiste à s'approprier le texte original et à le mettre au service de l'idéologie féministe s'accompagne, comme il fallait s'y attendre, d'une utilisation généreuse de préfaces, notes ou introductions. «It is becoming almost routine for feminist translators to reflect on their work in a preface, and to stress their active presence in the text in footnotes.» (L. von Flotow, 1991, p. 76) Leur adhésion à l'idéologie féministe les amène tout naturellement à se transformer elles aussi en pédagogues.

Il est frappant de constater à quel point les préfaces signées par les traducteurs du Moyen Âge et celles des traductri- 
ces modernes se ressemblent. Au-delà des analogies formelles, liées au genre de la préface, des rapprochements s'imposent. Les préfaces ont en commun de se développer selon un schéma analogue. Elles décrivent les principales difficultés de traduction rencontrées lors de l'interprétation ou de la restitution du texte original, incluent des rapprochements avec des faits contemporains ou d'autres écrits et, enfin, donnent des indications sur la portée des œuvres traduites et sur la manière de les lire. Voyons des exemples de chacun de ces points.

Schéma. Serge Lusignan (1986, pp. 131-132) a montré que les préfaces des traducteurs médiévaux se développent selon un schéma assez fixe:

a) Le mécène a beaucoup insisté auprès du traducteur pour qu'il prenne en charge le travail.

b) Le traducteur hésite, car il ne se sent pas à la hauteur de la tâche.

c) Il se fait prier et proposer des gratifications.

d) Il vante les mérites du commanditaire et de l'œuvre.

e) Malgré la faiblesse de ses moyens, il finit par accepter la tâche.

f) Il se dit honoré d'accomplir un travail utile pour le roi ou son seigneur.

g) Enfin et surtout, il ne manque pas de faire état des difficultés qu'il a rencontrées. Une double raison le motive à agir ainsi:

(i) répondre par avance à ses éventuels détracteurs;

(ii) solliciter l'indulgence de ses lecteurs.

Les préfaces des traductrices féministes renferment une séquence de «thèmes» comparables. Celle dont Marlene Wildeman orne sa traduction (la Lettre aérienne, de Nicole Brossard) correspond presque point par point au schéma ci-dessus.

a) On lui passe commande d'une traduction: «Maureene FitzGerald, managing editor at The Women's Press, asked me if I would like to translate La Lettre aérienne." 
b) Elle fait voir la difficulté de l'entreprise: «from the outset, the "specific" task proved to be constantly challenged by various practical and ethical questions» / «Brossard's work is not by nature accessible».

c) Elle doute un instant de ses capacités: «whether [...] I would be able to get this (collected) aerial vision off the ground."

d) Malgré la difficulté de la tâche, elle accepte, compte tenu de l'importance de l'œuvre: «I knew La Lettre in translation would have enormous impact on lesbian and feminist writers.»

e) Elle se dit honorée d'avoir été pressentie pour accomplir ce travail: «I felt honoured to have been asked to deliver it.»

f) Elle devance les critiques de ses éventuels détracteurs en montrant qu'elle est bien consciente des «translation dilemmas which posed ethical questions".

g) Elle sollicite l'indulgence des lecteurs en soulevant plusieurs questions du genre de celle-ci: «Is the translator ever forgiven for having temporarily abandoned the sustaining metaphor of a given fragment in the interest of continuity in the target language?»

La traduction par Howard Scott de l'ouvrage de Madeleine Gagnon, Antre, s'ouvre par une préface qui présente de fortes analogies avec celle de Marlene Wildeman.

a) Le traducteur hésite à s'attaquer à la traduction d'une œuvre féministe, d'autant plus qu'il est un homme. «I wondered if it was my place, as a man, to try to translate writing based so much on the subversion of traditional masculine discourse."

b) Il accepte néanmoins l'offre de traduire Antre. "when Maïr Verthuy [...] suggested I translate Antre, I took up the challenge.»

c) Il s'excuse à l'avance auprès de l'auteure et des lecteurs pour les imperfections de son travail. 
«I apologize to Madeleine and the reader for every time my translation falls short, either because I am a man, or for whatever other reason.»

d) Il confie avoir fait de son mieux malgré les énormes difficultés qu'il eut à surmonter.

«I have done my best to do justice to it [Antre] and to them [the readers].»

e) Il expose certains problèmes épineux de traduction. «This kind of writing presents special problems for the translator. I would like to briefly discuss two of those difficulties I encountered in Antre.»

f) Il se déclare seul responsable de la traduction, même si sa lecture de l'œuvre a reçu l'aval de l'auteure.

«I am solely responsible for the final version of this translation [...]. My re-reading of Antre was shared with Madeleine.»

Description des difficultés. Au-delà de tous les cas d'espèces analysés par les traducteurs médiévaux et les traductrices féministes dans leurs préfaces ou notes, on peut dire que ces deux groupes d'artisans de la traduction ont une conscience particulièrement aiguë des problèmes d'ordre linguistique. Leur rapport à la langue est problématique. Pour les premiers, ce sont les structures synthétiques du latin et l'indigence du lexique français savant qui constituent les principaux obstacles à vaincre. «No age was closer to, or more aware of, the linguistic problems involved in transforming Latin's synthetic structure into the analytic patterns of Romance.» (J. Beer, 1989, p. 3) La majorité des traducteurs sont frappés par l'impossibilité de traduire le latin à la lettre, «les constructions d'icellui [étant] si trenchies et si brieves, si suspensives et de si estranges mos».6 Tous auraient pu reprendre à leur compte cette parole de Laurent de Premierfait: «...ce qui semble trop brief je le allongeray, en exposant par mots et par sentences» (Ibid., p. 37).

6. Pierre Bersuire, cité par Paul A. Horguelin (1981), p. 31. 
La langue patriarcale présente des obstacles non moins grands aux traductrices féministes qui, faisant la chasse aux stéréotypes sexistes véhiculés par le langage, sentent elles aussi le besoin de faire état des stratégies mises en cuvre dans leurs traductions afin de décorseter la langue. Cette langue, jugée foncièrement misogyne, elles l'attaquent, la déconstruisent, en court-circuitent les formes sexistes, dévalorisantes, voire méprisantes pour les femmes. $\AA$ travers leurs explications et leurs commentaires, elles révèlent leur conception de la traduction et les règles qui les ont guidées dans leur travail de réécriture en langue d'arrivée.

C'est aussi dans un souci didactique que Pierre Bersuire fait précéder sa traduction des Décades de Tite-Live d'un lexique de 70 mots nouveaux qu'il lui a fallu créer pour compenser la pénurie de ressources lexicales du français d'alors. Son exemple sera imité par d'autres traducteurs, dont Jean Daunin et Nicole Oresme. De façon similaire, $c^{\prime} e s t$ souvent dans leurs préfaces que les traductrices d'aujourd'hui justifient les néologismes et autres créations discursives qu'elles inventent dans le but avoué de déranger les habitudes de lecture de tout un chacun et de ruser avec le masculin grammatical, afin de rendre compte de l'expérience féminine à travers la langue patriarcale.

Expérience personnelle et «intertexte». Les traducteurs de l'époque médiévale ne se font pas faute de procéder à des rapprochements avec des faits contemporains ni d'ajouter au texte original des exemples tirés de leur culture livresque ou de leur expérience personnelle. Ainsi, Simon de Hesdin (XIV s.) éprouve le besoin d'expliquer à son lecteur ce qu'est un «sesterce»: «Sexterce estoit certaine valeur de monnoie comme seroit en nostre commune maniere une livre de Paris ou de Tournois, mais nous ne savons mie certainement qu'elle valoit pource que les monnoyes, les pois et les valeurs sont trop variés des le temps Valerius jusques a present.. ${ }^{7}$ Nous donnerons plusieurs autres

7. Cité par Giuseppe di Stefano (1977), p. 58, note 26. 
exemples au point cinq ci-dessous de ce trait caractéristique des traductions de l'époque.

Les traductrices féministes aussi interviennent abondamment dans l'intertexte en multipliant les références aux écrits féministes. Susanne de Lotbinière-Harwood, par exemple, ponctue sa traduction Letters From An Other (Lettres d'une autre, $\mathrm{N}$. Brossard) de 135 notes. Le texte imprimé comptant 143 pages, cela représente tout près d'une intervention par page en moyenne. Ces notes sont de diverse nature: encyclopédiques, traductionnelles, linguistiques, culturelles, historiques, sociologiques, idéologiques. Et comme si cela ne suffisait pas, s'y ajoutent une préface de Sherry Simon et une introduction de la traductrice. Dans Re-belle et infidele, S. de Lotbinière-Harwood écrit:

[...] les notes de la traductrice entrent dans la composition de l'intertexte féministe. Elles attestent de l'ampleur et de la richesse de la production littéraire des femmes. Il en va de même pour les préfaces, qui sont également un bon moyen d'amener le public lecteur à mieux comprendre et apprécier la traduction. (pp. 46-47)

Des articles publiés dans diverses revues poursuivent la réflexion amorcée dans les préfaces et traitent des mêmes sujets.

Portée de l'œuvre traduite. Véritables précepteurs des rois, les traducteurs du Moyen Âge utilisent leurs préfaces pour préciser l'optique dans laquelle il convient de lire les textes anciens. La lecture des œuvres de l'Antiquité gréco-romaine doit fournir au roi et aux seigneurs commanditaires des modèles à imiter. Les traducteurs prennent même la liberté de mettre en garde les têtes dirigeantes du royaume contre les erreurs commises par un Alexandre ou un César, qui n'ont pas su freiner leurs ambitions et sont tombés dans la démesure.

Les traductrices contemporaines n'agissent pas autrement. Leurs textes liminaires insistent sur la portée féministe des œuvres (originales et traduites) et indiquent au lecteur dans quel esprit il faut les lire. Dans la préface qu'elle rédige pour présenter sa traduction d'Amantes, Barbara Godard renseigne le lecteur sur 
le sens de l'œuvre de Nicole Brossard et lui donne des indications sur la façon dont il convient de la lire et de la comprendre. Elle fait de même pour sa traduction de l'ouvrage de France Théoret, le Mot tangible. Cette pratique est courante chez les traductrices féministes. Toutes ces indications sur le sens des œuvres traduites ne procèdent pas uniquement de préoccupations d'ordre littéraire. Elles sont aussi et surtout des manifestations concrètes d'un militantisme féministe qui porte spontanément les traductrices à amplifier l'aspect didactique de leur travail: «[...] a feminist translation becomes an educational tool supported with scholarly research", constate Luise von Flotow (1991, p. 77).

D'ailleurs, presque invariablement, l'œuvre traduite est présentée comme une traduction féministe. D'entrée de jeu, la traductrice annonce ses couleurs. Susanne de Lotbinière-Harwood écrit au début de l'introduction dont elle fait précéder sa traduction de Lettres $d^{\prime}$ 'une autre de Lise Gauvin: «Just a few words to let you know that this translation is a rewriting in the feminine of what I originally read in French.» (S. de LotbinièreHarwood, 1989, p. 9; c'est nous qui soulignons.) Pour sa part, Marlene Wildeman (1988), qui se définit elle-même comme "a Canadian feminist writer and translator", affirme:

When I began translating this book I found myself in a very privileged position with a specific task at hand and clear feminist obligations: translate Nicole Brossard's La Lettre aérienne for English feminist readers, and in the process, create a certain English lesbian feminist perspective on Nicole Brossard, [...]. (p. 27; c'est nous qui soulignons.)

\section{Interventions sur la langue}

«Une langue est une prison. La posséder, c'est l'agrandir un peu.» Cette réflexion de l'écrivain et traducteur québécois Pierre Baillargeon (1947, p. 131) s'applique bien aux deux groupes de traducteurs qui nous occupent ici. Les traducteurs médiévaux, avons-nous vu, ont conscience des différences énormes qui séparent les moyens d'expression du latin et ceux du français d'alors. Il leur faut passer d'une langue littéraire pleinement 
constituée (le latin) à une langue encore pauvre et en voie d'acquérir sa maturité sur le plan de l'écriture. «ll s'agissait pour les traducteurs non seulement de traduire, mais de créer jusqu'à un certain point la langue cible dans laquelle ils devaient incruster l'œuvre latine. [Ils] opère[nt] sur la langue tout autant que sur le texte.» (S. Lusignan, 1986, p. 149; c'est nous qui soulignons.) L'acte de traduire à l'époque médiévale est un gigantesque effort pour doter le français de tous les moyens d'expression, de tous les registres d'une grande langue de civilisation. Pour passer de "fort latin" à "clair et entendable romant», selon l'expression, maintes fois citée, de Simon de Hesdin, les traducteurs médiévaux n'ont pas hésité à mettre en œuvre plusieurs moyens: calque, emprunt, périphrase, etc. Ils ont aussi fécondé la langue d'une multitude de néologismes, contribuant ainsi à rendre le français apte à exprimer des abstractions. Ils ont été des artisans de l'assouplissement de la syntaxe française. Le lexique de Pierre Bersuire, évoqué plus haut, renferme plusieurs créations néologiques qui sont aujourd'hui d'un usage courant: «augure», «cirque», «expié», "transfuge", "fonction", "sénat», "triomphe». Pour sa part, Nicole Oresme aurait introduit dans la langue française pas moins de 450 mots nouveaux, selon Robert Taylor (1965). Ce chiffre ne tient compte que des néologismes encore présents dans le français contemporain: «anarchie», "architecte», "comédie», «législatif», "pédagogue», «agent», «réflexion», «total». «Quantité de mots [ont été] forgés sur l'enclume de la traduction médiévale.» (Lusignan, 1986, p. 165)

Ce façonnage de la langue amène aussi les traducteurs à distinguer les nuances de sens entre deux termes. Le redoublement de mots était un procédé commun pour préciser le sens d'un terme nouveau ou technique: «le pris et la somme de rachat», "selon la manière et coutume du païs", "en une ambulation ou aler». Par ces doublets, qui subsisteront dans la langue jusqu'au $\mathrm{XVI}^{e}$ siècle, le traducteur manifeste son souci d'être compris. Ces stratégies de traduction ont eu l'effet d'un extraordinaire travail de pétrissage de la langue qui a ouvert la voie au français classique. 
Le remodelage du lexique et des formes discursives auquel s'adonnent les féministes en général et les traductrices d'allégeance féministe en particulier procède d'une démarche tout à fait comparable à celle des traducteurs d'autrefois. «Inventiveness, playfulness, subversion become the tools of the feminist translator as well as of the feminist writer.» (K. Mezei, 1989, p. 10) Confrontées aux formes jugées sexistes de la langue tout comme les traducteurs médiévaux à la grande «briefté» (concision) du latin, les traductrices cherchent à inventer un nouveau langage reflétant explicitement la présence active des femmes dans la société. Howard Scott (1989) a exprimé en termes fort clairs l'action militante des féministes québécoises par le biais de leurs interventions sur la langue, et ce qu'il dit des théoriciennes vaut tout autant pour les traductrices anglophones de leurs œuvres:

They work words in different ways, in subversive ways, disrupting the linearity of conventional discourse, deconstructing grammar, sabotaging the symbolism of patriarchy, stripping words to their bare meanings and breaking open language to let it say what is unsaid and unsayable in the language of patriarchy. Through these linguistic transgressions, they expand cultural space to liberate territory for women's expression. (p. 6)

Les stratégies d'écriture et de traduction appliquées par les féministes sont aussi variées que celles de leurs lointains prédécesseurs. Les traductrices procèdent à l'autopsie des mots, scrutent leur étymologie afin de les déconstruire. Isoler un préfixe de son radical est une pratique courante chez elles. On ne compte plus également les néologismes créés dans le sillage du mouvement féministe, notamment dans le domaine de la désignation de fonctions, de métiers ou de professions. Qu'il suffise de citer les spokesperson, salesperson, chairperson, businessperson, Ms., etc. et, pour le français, les «écrivaine», «factrice», "agente", "cheffe", "pompière", "professeure", etc. Dans le domaine de l'esthétique littéraire, la création néologique donne parfois lieu à des trouvailles étonnantes relevant du ludisme langagier: loohers (lovers - B. Godard), herstory (history), outsid(hlers, «ellogiquement» ou «il-logique» (S. de Lotbinière-Harwood), 
«scribelle» (féminin de scribe - N. Brossard). Les féministes ont réintroduit aussi des mots tombés en désuétude comme «cyprine», terme désignant les "sécrétions sexuelles des femmes» (S. de Lotbinière-Harwood, 1991, p. 59). Le redoublement de certains pronoms ou possessifs (ceux et celles, his/her, she/he), de certains titres de fonction ou de profession (directeur/directrice, avocat/avocate), de certains collectifs (les étudiants et les étudiantes, les citoyens et les citoyennes) n'est pas sans rappeler les doublets des traducteurs du Moyen Âge. Certaines marques typographiques (gras, parenthèses, obliques) sont aussi utilisées judicieusement pour faire ressortir la double signification de certains mots. Comme l'ont fait leurs prédécesseurs Nicole Oresme, Jean de Meung et d'autres, les traductrices féministes procèdent à de minutieuses distinctions de sens. Les mots «jouir» et «jouissance» se révèlent particulièrement difficiles à traduire en anglais, car ils ne recouvrent pas tout à fait, semble-t-il, la signification de pleasure. En somme, pour agrandir quelque peu la prison que constitue la langue, les traducteurs médiévaux et les traductrices contemporaines, opérant autant sur la langue que sur le discours, ont mis en cuvre des stratégies assez similaires: périphrases, néologismes, doublets, emprunts, etc. $\AA$ une similitude des fins poursuivies correspond une similitude de moyens.

\section{Visibilité du traducteur ou de la traductrice dans sa traduction}

Nous avons vu que les traducteurs médiévaux tout comme les traductrices féministes font sentir leur présence dans leurs traductions en les assortissant de préfaces et de notes. Le lecteur sent constamment le traducteur ou la traductrice à l'œuvre entre lui et l'auteur. Cela est tout particulièrement manifeste chez les traducteurs médiévaux qui usaient abondamment de gloses, de commentaires et d'interpolations. Ils restent près de leurs lecteurs à qui ils s'adressent même directement à l'occasion. Mathieu le Vilain (XIII s.) signale à Jean, comte $d^{\prime} E u$, les difficultés du texte qu'il traduit à son intention. Il insère dans sa traduction des commentaires du genre: «Or semble, sire comte, que ceste parole soit contraire à ce qu'il avoit dit devant... Mais ce n'est pas 
contrariété. Car...» ${ }^{8}$ Jean de Meung (v. 1250-1305), lui, émaille sa traduction $\mathrm{d}^{\prime}$ Abélard et Héloise de réflexions personnelles dont certaines ne manquent pas de piquant. Tantôt il approuve ce que dit l'auteur: «Nota: onques [jamais] femme ne parla plus sagement", tantôt il cite un proverbe qui lui paraît bien s'appliquer à la situation: «Et ce s'accorde à ceste parole: qui premier est coux [cocu] en la ville, derrenier le scet.» (Ibid., p. 561) Il arrive aussi que le traducteur identifie le lecteur avec l'auteur: «Cypre, chascun scet que c'est.." Confronté à un problème de désignation, le traducteur avouera candidement son impuissance et déclarera forfait. Simon de Hesdin, par exemple, incorpore dans sa traduction de fréquentes interpolations comme celles-ci: «...l'autre royaume estoit appelé siculum en latin que je ne scay dire en rommant..."; "Les peuples ou pays nomme Orose ou Ve livre ou $\mathrm{XVIII}^{e}$ chappitre, et pource que je ne le say proprement mettre en romant, je les nomme en la maniere comme il les nomme...»; à propos du mot socer, il écrit: «pour lequel je n'ay point de propre terme en françois.» (Ibid., p. 52; c'est nous qui soulignons.) Le traducteur ne s'efface donc pas totalement devant l'auteur. Il est un médiateur apparent, une sorte de présentateur, de commentateur de l'œuvre ancienne qu'il traduit. Cette présence se manifeste aussi par l'information de nature encyclopédique que le traducteur ajoute au texte et par de fréquents renvois a ses connaissances livresques ${ }^{10}$.

Les traductrices laissent aussi de nombreuses traces d'elles-mêmes dans leurs traductions. Elles n'hésitent pas à creuser l'écart existant entre le texte original et sa version

8. Cité par Paul Chavy (1974), p. 560.

9. Cité par Giuseppe di Stefano (1977), p. 66.

10. Dans son "prohesme», Simon de Hesdin justifie ses interventions en ces termes: "Valerius ne peut pas de tout avoir memoire et, pour ce, je veul à la fois mettre aucuns exemples qui ne sont point en ce livre, quant il me sembleront propres a la matiere duquel Valerius parle.» Cité par Paul Chavy (1988), p. 1313. 
traduite. Susanne de Lotbinière-Harwood (1991) propose de nombreux moyens «pour faire acte de présence dans le texte traduit» et "placer sa voix par rapport à celle de l'auteur-e» (p. 26). Les moyens préconisés sont largement mis en pratique par toutes les traductrices féministes: doublets constitués de la forme masculine et féminine [heroes and heroines], parenthèses [while they (men)], trait d'union [Québécois-e], guillemets ["les hôtesses» ${ }^{11}$, barres obliques [her/his; s/he], etc.

La traductrice envahit le texte de sa présence, de sa présence féminine, par l'emploi de stratégies qui ne coïncident pas forcément avec celles appliquées par l'auteure du texte de départ. Ce sont autant de traces ostensibles de la «main traduisante». La traductrice sort de l'ombre. "No longer is the translator silent behind the text.» (Mezei, 1989, p. 10) La traductrice fait tout pour que le lecteur n'oublie pas qu'il tient une traduction entre ses mains. Susanne de Lotbinière-Harwood (1989) le prévient même explicitement: «You won't be able to forget that this is a translation.» (p. 9)

Les traducteurs médiévaux et les traductrices féministes refusent donc de s'effacer devant l'auteur, de n'être que des «fantômes de service». Ils et elles prennent la parole et produisent ce qu'on peut appeler des traductions-commentaires, les premiers dans un souci évident de mettre les œuvres anciennes à la portée de leurs lecteurs, les secondes dans un désir de promouvoir l'idéologie féministe. Dans un cas comme dans l'autre, nous sommes bien loin des timides «En français dans le texte. (N.D.T.)» que s'autorisent les traducteurs littéraires modernes.

Enfin, les traducteurs et traductrices de ces deux époques sont aussi présents «visuellement» dans leur texte. Les premiers dans des miniatures, où ils sont représentés habituellement agenouillés devant leur mécène à qui ils remettent leur traduc-

11. "...to highlight the inadequacy of male-made language for speaking in the feminine» (S. de Lotbinière-Harwood, 1989, p. 12). 
tion. Les secondes sont photographiées en compagnie de l'auteure traduite, parfois assises à une même table de travail, devant deux tasses de café. La déférence des premiers à l'égard du mécène fait place chez nos contemporaines à une posture plus égalitaire, plus conforme à l'image qu'elles ont d'elles-mêmes: celle de coauteures de l'œuvre originale.

Les textes de présentation des auteures et de leurs traductrices figurant en fin de volume ou en quatrième de couverture sont aussi révélateurs de ce statut égalitaire. Ils sont sensiblement de même longueur et apportent autant de renseignements bio-bibliographiques sur la traductrice que sur l'auteure. Une fois de plus, on est loin de la simple mention en petits caractères du nom du traducteur sur la page titre des œuvres traduites, comme c'est habituellement le cas.

\section{Conclusion}

Le rapprochement que nous venons d'effectuer entre la manière de traduire à deux époques distantes de plus de cinq cents ans nous révèle que la façon de traduire est historiquement déterminée. Les traducteurs médiévaux avaient conscience de contribuer à faire perdre au latin son statut dominant de langue savante; les traductrices féministes, pour leur part, remettent en cause le "parler-homme» dominant qui occulte la présence du «deuxième sexe». Dans les deux cas, il s'agit de briser un monopole.

Pour jouer ce rôle de médiateur actif, les traducteurs et les traductrices sont amenés à se donner une visibilité accrue et cessent d'être des "quasi-abstractions", des "mains invisibles», des "voix silencieuses» au service d'un auteur ou d'une auteure. Cela ne va pas sans une nécessaire appropriation du texte original, qui devient en quelque sorte le véhicule de leurs convictions personnelles. Il en résulte une accentuation du caractère didactique de leur travail et un désir manifeste de se rapprocher du lecteur, de guider ses pas sur un territoire encore mal cartographié. 
Les deux cas évoqués ne sont pas les seuls exemples d'une stratégie de prise de parole de la part de traducteurs. L'histoire de la traduction nous en fournit de nombreux autres. Par exemple, sous le régime communiste de l'ex-URSS, "privés de la possibilité de s'exprimer totalement dans une cuvre originale, les poètes russes [surtout entre 1934 et 1956] s'entretenaient avec le lecteur par le truchement de Gœethe, de Shakespeare, d'Orbéliani et de Hugo».12 Et que dire des traducteurs québécois qui, suivant le courant de la nouvelle dramaturgie, la montée du nationalisme au Québec et l'avènement d'une langue qui se voulait typiquement "québécoise», distincte de la française, ont naturalisé le théâtre étranger en faisant parler à la québécoise les personnages de Shakespeare, Tchekhov, O'Neill, Lorca, Brecht et Goldoni. A travers leurs traductions, les adaptateurs s'employaient à proclamer le fait québécois au lieu de servir l'œuvre étrangère ${ }^{13}$.

Des détournements de ce genre exigent une complicité. Celle-ci s'établit au Moyen Âge entre le traducteur et le lecteur, et aujourd'hui entre la traductrice, le lecteur et les auteures traduites. Ces dernières collaborent d'ailleurs aux traductions et donnent leur aval aux prises de position militantes de leurs traductrices avec qui elles sont unies dans un même combat. "Complicity is the nature of the relationship between translator and writer in such translations, when translation becomes creation but also subversion. ${ }^{14}$

12. Efim Etkind (1977), p. 50. Cette phrase valut à son auteur, théoricien de la traduction et réputé traducteur de poésie, d'être relevé de ses fonctions, privé de ses titres universitaires et exclu de l'Union des écrivains.

13. Voir A. Brisset (1990).

14. B. Godard (1982), p. 36. S. de Lotbinière-Harwood (1991) écrit pour sa part: «Il est essentiel de travailler avec l'auteure à la préparation du manuscrit de la traduction. Cela fait partie intégrante du processus. En plus de développer une complicité littéraire active entre femmes, cette collaboration occasionne 
Enfin, la manière de traduire que nous avons décrite offre un bel exemple de mariage de théorie et de pratique de la traduction. Les obstacles à surmonter amènent sans cesse les traducteurs et traductrices à réfléchir aux solutions et à fonder leurs choix sur un ensemble cohérent de principes, de règles et de procédés de traduction. Ces réflexions issues de la pratique enrichissent en retour la théorie de la traduction, tout comme les néologismes des traducteurs médiévaux ont fécondé la langue et enrichi son vocabulaire ${ }^{15}$. Les traductrices qui osent prendre la parole et assortir leurs travaux de préfaces, de notes et de commentaires sur l'art de la lecture et sur cette forme particulière de réécriture qu'est la traduction nous forcent à nous interroger sur la substance du sens et sur les notions de fidélité, de transparence et d'équivalence de deux textes. La façon dont elles conçoivent la fidélité coïncide avec les théories contemporaines de la traduction qui ne voient plus la recherche des équivalences en traduction comme une recherche d'identité. Lorsqu'elle pratique la traduction-appropriation, la traductrice procède à une opération de remaniement de l'œuvre originale. «[...] le sens d'un texte est négociable.» (G. Scott, 1985, p. 5) Elle s'arroge, mais avec le consentement de l'auteure, les prérogatives de celle-ci. Il en résulte une œuvre à la fois semblable et autre. La nouvelle œuvre englobe et "cache" l'originale. En réintroduisant en force la subjectivité dans l'acte de traduire, les traductrices féministes définissent une nouvelle éthique de la traduction. Mais est-ce vraiment une nouvelle éthique? Les traducteurs français ne la pratiquaient-ils pas déjà à l'époque des bâtisseurs de cathédrales?

Université d'Ottawa

souvent des découvertes aussi inattendues qu'extraordinaires.» (p. 71)

15. Cf. G. Mounin (1963), chap. XII: «Les Universaux du langage», pp. 191-223. 


\section{Références}

BAILLARGEON, Pierre (1947). Commerce. Montréal, Les Éditions Variétés, $185 \mathrm{p}$.

BEER, Jeanette, dir. (1989). Medieoal Translators and their Craft. Kalamazoo, Western Michigan University, 428 p.

BERSIANIK, Louky (1976). L'Euguélionne. Montréal, Éditions La Presse, $400 \mathrm{p}$.

BRISSET, Annie (1990). Sociocritique de la traduction. Thêâtre et altérité au Québec (1968-1988). Montréal, Le Préambule, $347 \mathrm{p}$.

BROSSARD, Nicole (1977). L'Amèr ou le chapitre effrité. Montréal, Éditions Quinze.

(1985). La Lettre aérienne. Montréal, Les Éditions du Remue-Ménage.

CHAVY, Paul (1974). «Les Premiers translateurs français», The French Review, vol. XLVII(3), pp. 557-565.

(1989). «Domaines et fonctions des traductions françaises à l'aube de la Renaissance», Revue de littérature comparée, 250, pp. 147-153.

(1981). «Les Traducteurs humanistes au début de la Renaissance française: traductions médiévales, traductions modernes", Reoue canadienne de littérature comparée, vol. VIII(2), pp. 284-306.

(1988). Traducteurs d'autrefois. Moyen Âge et Renaissance. Dictionnaire des traducteurs et de la littérature traduite en ancien et moyen français (842-1600). Paris/Genève, les Éditions Champion-Slatkine. Vol. I (A-J), pp. 1-810; vol. II (K-Z), pp. 811-1544. 
DI STEFANO, Giuseppe (1977). Essais sur le moyen français. Padoue, Liviana Éditrice, $139 \mathrm{p}$.

DU BELLAY, Joachim (1549). Deffence et illustration de la langue francoyse. Édition critique de Henri Chamard: Paris, Éditions Didier, 1966, 206 p.

ETKIND, Efim (1977). Dissident malgré lui. Trad. du russe par Monique Slodzian. Paris, Éditions Albin Michel, 315 p.

FLOTOW, Luise von (1991). «Feminist Translation: Contexts, Practices and Theories», TTR, IV(2), pp. 69-84.

GAUVIN, Lise (1987). Lettres d'une autre. Montréal, Éditions L'Hexagone, 149 p.

(1989). Letters From An Other. Trad. par Susanne de Lotbinière-Harwood. Toronto, The Women's Press, 143 p.

GODARD, Barbara (1983). «Preface», These Our Mothers or: The Disintegrating Chapter (N. Brossard, L'Amèr ou le chapitre effrité). Toronto, Coach House Press.

(1989). «Theorizing Feminist Discourse/ Translation», Tessera 6 («La Traduction au féminin / Translating Women»), printemps, pp. 42-53.

(1984). «Translating and Sexual Difference», Resources for Feminist Research, vol. XIII(3), pp. 13-16.

(1986). «Preface», Lovhers (N. Brossard, Amantes). Trad. par B. Godard. Montréal, Éditions Guernica, pp. 7-12.

(1982). «The Translator as Ventriloquist», Prism International, vol. $\mathrm{XX(3),} \mathrm{pp.} \mathrm{35-36.}$ 
(1992). «Translating Translating Translation» (Preface), The Tangible Word (France Théoret, le Mot tangible), pp. 7-15.

HOMEL, David et Sherry SIMON, dir. (1988). Mapping Literature. The Art and Politics of Translation. Montréal, Véhicule Press, $127 \mathrm{p}$.

HORGUELIN, Paul A. (1981). Anthologie de la manière de traduire. Domaine français. Montréal, Linguatech, 230 p.

LOTBINIËRE-HARWOOD, Susanne de (1991). Re-Belle et Infidèle. La traduction comme pratique de réécriture au féminin / The Body Bilingual. Translation as a Rewriting in the Feminine. Montréal/Toronto, Les Éditions du Remue-Ménage / The Women's Press, 175 p.

(1989). "About the her in other», Letters From An Other (N. Brossard, Lettres d'une autre), pp. 9-12.

LUSIGNAN, Serge (1986). Parler vulgairement. Paris/Montréal, Vrin/Presses de l'Université de Montréal, 204 p.; chap. IV: «Le mouvement des traductions au XIV siècle et la définition du français comme langue savante».

MEZEI, Kathy (1989). «Traverse», Tessera 6, («La Traduction au féminin / Translating Women»), printemps, pp. 9-10.

MONFRIN, Jacques (1964a). «Humanisme et traduction au Moyen Âge», Actes du colloque organisé par le Centre de Philologie et de Littératures romanes de l'Université de Strasbourg, publiés par Anthème Fourrier. Paris, C. H. Klincksieck, pp. 217-246.

(1964b). «Les Traducteurs et leur public en France au Moyen Âge», Actes du colloque organisé par le Centre de Philologie et de Littératures romanes de l'Université de Strasbourg, publiés par Anthème Fourrier. Paris, C. H. Klincksieck, pp. 247-262. 
MOUNIN, Georges (1963). Les Problèmes théoriques de la traduction. Paris, Éditions Gallimard, 297 p.

RICARD, François (1992). La Génération lyrique. Montréal, Éditions Boréal, 282 p.

SCOTT, Howard (1989). «Translator's Introduction», Lair (Madeleine Gagnon, Antre), pp. 5-10.

SCOTT, Gail (1988). Héroïne. Trad. par Susanne de LotbinièreHarwood. Montréal, Éditions du Remue-Ménage, 250 p.

(1985). «Liminaire», Tessera 2 («L'écriture comme lecture»), pp. 5-6.

SIMON, Sherry (1989). «Preface», Letters From An Other (L. Gauvin, Lettres d'une autre), pp. 5-8.

SMART, Patricia (1988). Écrire dans la maison du père. L'émergence du féminin dans la tradition littéraire du Québec. Montréal, Québec/Amérique, 337 p.

TAYLOR, Robert (1965). «Les Néologismes chez Nicole Oresme, traducteur du XIV ${ }^{e}$ siècle», Actes du $X^{e}$ congrès international de linguistique et philologie romanes, publiés par Georges Straka. Paris, Klincksieck, t. II, pp. 727-736.

TESSERA 2 (1985), “L'Écriture comme lecture», Nbj 157, 100 p.

TESSERA 6 (1989). «La Traduction au féminin / Translating Women", printemps, Simon Fraser University, Burnaby, Dept. of English, 96 p.

WILDEMAN, Marlene (1989). «Daring Deeds: Translation as Lesbian Feminist Language Act», Tessera 6 («La Traduction au féminin / Translating Women"), printemps, pp. $31-40$. 
(1988). "Translator's Introduction», The Aerial Letter (N. Brossard, la Lettre aérienne), pp. 27-31. 